strong sulphuric acid, and a knife was placed in the filtrate. At the end of eighteen hours the knife showed, to the extent of its immersion, two inches, a complete covering of copper on both sides. The evidence of experiments 1,2 , and 3 was shown in court and their significance explained to Sheriff Birnie, who tried the case at the instance of the sanitary suthority of Glasgow. The evidence for the prosecution and the defence is fully and correctly given in the Chemist and Druggist, in its issues of Jan. lst and Jan. 15th. A carefnl reading of each shows that the evidence for the defence in no way traversed what we had done, and which was supplemented by such well-known chemists as Messrs. Tatlock and Clarke. Yet, in deciding for the defence, the Sheriff stated that "no new evidence had been brought forward." By his decision he sanctioned the use of a strong irritant metallic poison in food intended for consumption by human beings, the amount of which is not fixed, but is left to the discretion of foreign manufacturers over whom the authorities of our country have no control.

Glasgow.

$O N$

\section{TWO CASES OF ANTHRAX SUCCESSFULLY TREATED BY EXCISING THE PUSTULE.}

BY T. PAGAN LOWE, M.R.C.S.,

HONORARY MEDICAL OFFICER TO THE EASTERX DISPENSARY, BATH.

THESE two cases occurred in March of last year. This is the first time, so far as I am aware, that the disease has been known in Bath.

A man named W-, aged thirty-eight, was first attacked, his wife contracting the disease fourteen days later. I saw him for the first time on March 11th, the seventh day of the disease. I found him suffering from a large malignant pustule on the right side of the neck, about the middle of the posterior triangle. The pustule consisted of a central dark-brown eschar, surrounded by a zone of flattened vesicles, outside of which was an inflammatory zone, the whole of the right side of the neck being enormorsly swollen and cedematoas. The treatment $I$ adopted was that of removing the purstule by an elliptical incision; and this included the whole area of vesiculation, together with a considerable portion of healthy skin on each side and the deep tissues beneath, so as to ensure complete eradication. The wound was freely irrigated with hot perchloride solution, and well dusted with iodoform before its margins were brough together by sulures. The patient made a satisfactory and uninterrupted recovery. Mr. Arthur Barker kindly examined the specimen and confirmed the diagnosis.

Mrs. $W$ - the wife, aged forty, who was assiduous in her attentions upon her husband, and to whom were entrusted the evening dressings, was attacked by the same disease fourteen days later. In her case the pustule appeared on the cheek. In the absence of proper home nursing I was obliged to send the case into hospital, where the pustule was excised by Mr. Stockwell, and its base frealy cauterised. The operation in this case was performed on the fourth day, and was followed by rapid convalescence. In both instances the disease was inoculated by the finger nail, the husband having scratched his neck after washing some buffalo hide, and his wife her cheek after dressing her husband's wound. Both cases suggest that anthrax, when it attacks the skin, may remain local for a considerable time, and produce a mild affection as compared with the same disease when attacking internal organs. The bacilli of anthrax are known to have a marked preference for the superficial layers of the dermis, slowly penetrating into the deeper parts. Nevertheless, it seems very extraordinary that, in the case of anthrax the excision of the focus of inoculation shonld arrest the progress of the disease. It may be explained on the hypothesis that the behaviour of the tissues towards the bacillus is very different from that towards most other infective micro-organisms, and an illustration is obtained of one form of tissue resistance. Very valuable information may yet be gleaned from observing more closely the strange sequence of events. One fact may be claimed as having been well established-i.e., that theoretical considerations should never deter anyone from

A paper read before the Bath and Bristol Branch gof the British Yedical Association, Oct. 28th, 1891. operating, not only during the early stages, but at whatever period of the disease the cases present themselves. In fact, we have on record an ample number of instances in which complete success has followed excision during the later stages. It seems, noreover, that we have in the pustule a manufactory which supplies bacilli in unlimited quantities, and that, when this manufactory is removed, the microphages are well able to cope successfully with any organisms which may have escaped into the blood stream. The bacilli anthracis, although offering powerful resistance to cold and to strong solutions of carbolic acid, are quickly destroyed by low degrees of heat, a temperature of $120^{\circ} \mathrm{F}$. being amply sufficient for the purpose. The disease in many localities is sufficiently common and dangerous to warrant legislative interference to prevent the importation of hides, except on the condition that, prior to distribution, they undergo a process of heating at, say, $120^{\circ} \mathrm{F}$., for a given length of time. This process, whilst it would effectually stamp out the disease, would in no respect prejudice the commercial interest involved.

Bath.

\section{Clinital a dotes:}

MEDICAL, SURGICAL, OBSTETRICAL, AND THERAPEUTICAL.

\section{RUPTURE OF THE DUODENUM.}

\section{BY R. HEELIS, M.D. DURH., L.R.C.I.I.}

RUPTURE of the duodenum is said by Mr. Morris in Treves's Surgery to be of rare occurrence. Out of sixtyfour recorded cases of ruptured gut only four were in that situation. Erichsen, on the other hand, considers it the most common position for a rupture, and accounts for this, first, by the fixity of that part of the gut and its consequent inability to escape pressure against the spine; and, secondly, by the fact of its not being protected by the costal cartilages. Bryant says rupture of the duodenum is very rare, but that it is most common at the upper end of the jejunum, on account of its fixture to the duodenum. In view of this diversity of opinion, a report of a case of rupture of the duodenum which I have recently examined may be interesting.

On July 23rd, about 3.30 P.M., a boy aged sixteen years, employed at a neighbouring colliery, whilst playing about on the bank was caught between a post and a tub drawn by a horse, the side of the tub hitting him in the right loin and forcing his abdomen against the post. In his belt he wore a bull's-eye lantern which caught against the post and was doubled up by the force of the collision, and driven against his abdomen and right groin. He fell down screaming with pain, and after having been given some brandy. and-water was driven home. I did not see him until three or four bours after the accident, when $I$ found him lying in bed on his left side with his legs drawn up, groaning. $\mathrm{He}$ was not collapsed, and had a steady pulse; the abdomen was rigid and concave; there was a slight swelling in the right groin; no bruise in the loin or over the abdomen. He had vomited once before $I$ saw him, there was mo blood in the vomit; he had also passed urine, which did not contain blood. The principal tenderness was just above the right groin. He was given full doses of opium, under which the pain disappeared to a great extent.-July 24 th: Had passed a fuirly good night, but had vomited once or twice. On my visit I was surprised to find him downstairs, and still more so to hear that he had walked down. His pulse was good; his abdomen not distended; chief tenderness above the right groin as yesterday. Vomited bilious matter during the day, but ceased vomiting after he had had some ice. Dring the following night he died comatose, thirby-seven hours after the receipt of the injury.

Necropsy, thirty hours after death.-Advanced decomposition. The abdomen was greatly distended; there were gas in the abdominal cavity and some extravasation of blood between the muscles in the righoloin. The peritoneal cavity contained a small quantity of bloody serous fluid and the intestines were not adherent or lymph-coated. The small intestines below the duodenum were removed in order to 